\title{
Auswärtige Kulturpolitik
}

\section{Herausgegeben von}

Prof. Dr. Wolfgang Schneider 
„Die Pflege der Beziehungen zu auswärtigen Staaten ist Sache des Bundes“, heißt es in Artikel 32 des Grundgesetzes der Bundesrepublik Deutschland. Die „Pflege“ geschieht durch Diplomatie und wirtschaft liche „Beziehungen“. Dritte Säule der Außenpolitik ist die Auswärtige Kulturpolitik, die es zu untersuchen gilt. Dialog und Austausch mittels Kunst und Kultur sind Gegenstände von Politikwissenschaft und Kulturwissenschaft en. Studien der Kulturpolitikforschung analysieren und reflektieren Anspruch und Wirklichkeit von Projekten und Programmen der so genannten Mittlerorganisationen. Die von Wolfgang Schneider herausgegebene Reihe bei Springer fundiert Theorie und Praxis Auswärtiger Kulturpolitik auf nationaler Ebene, im komparatistischen internationalen Diskurs und im Rahmen der europäischen Integration. 
Wolfgang Schneider • Anna Kaitinnis (Hrsg.)

\section{Kulturarbeit in \\ Transformationsprozessen \\ Innenansichten zur ,Außenpolitik' des \\ Goethe-Instituts}

黑 SpringerVS 
Herausgeber

Wolfgang Schneider

Hildesheim, Deutschland
Anna Kaitinnis

Hildesheim, Deutschland

Auswärtige Kulturpolitik

ISBN 978-3-658-13258-3

ISBN 978-3-658-13259-0 (eBook)

DOI 10.1007/978-3-658-13259-0

Die Deutsche Nationalbibliothek verzeichnet diese Publikation in der Deutschen Nationalbibliografie; detaillierte bibliografische Daten sind im Internet über http://dnb.d-nb.de abrufbar.

\section{Springer VS}

(C) Springer Fachmedien Wiesbaden 2016

Das Werk einschließlich aller seiner Teile ist urheberrechtlich geschützt. Jede Verwertung, die nicht ausdrücklich vom Urheberrechtsgesetz zugelassen ist, bedarf der vorherigen Zustimmung des Verlags. Das gilt insbesondere für Vervielfältigungen, Bearbeitungen, Übersetzungen, Mikroverfilmungen und die Einspeicherung und Verarbeitung in elektronischen Systemen.

Die Wiedergabe von Gebrauchsnamen, Handelsnamen, Warenbezeichnungen usw. in diesem Werk berechtigt auch ohne besondere Kennzeichnung nicht zu der Annahme, dass solche Namen im Sinne der Warenzeichen- und Markenschutz-Gesetzgebung als frei zu betrachten wären und daher von jedermann benutzt werden dürften.

Der Verlag, die Autoren und die Herausgeber gehen davon aus, dass die Angaben und Informationen in diesem Werk zum Zeitpunkt der Veröffentlichung vollständig und korrekt sind. Weder der Verlag noch die Autoren oder die Herausgeber übernehmen, ausdrücklich oder implizit, Gewähr für den Inhalt des Werkes, etwaige Fehler oder Äußerungen.

Redaktion: Aron Weigl

Gedruckt auf säurefreiem und chlorfrei gebleichtem Papier

Springer VS ist Teil von Springer Nature

Die eingetragene Gesellschaft ist Springer Fachmedien Wiesbaden GmbH 


\section{Inhalt}

Abkürzungsverzeichnis ............................................................................

Auswärtige Kulturpolitik. Beiträge zu Theorie und Praxis binationaler

Beziehungen .............................................................................................

Wolfgang Schneider und Anna Kaitinnis

\section{Zur gesellschaftlichen Rolle von Kunst und Kultur}

Schnittmengen und Zielkonvergenzen. Überlegungen zur Auswärtigen

Kulturpolitik aus der Sicht der Demokratieforschung

Marianne Kneuer

Transformationsprozesse als Gegenstand von Kulturpolitikforschung.

Beobachtungen in Afrika

Wolfgang Schneider

,Tahrir ${ }^{\star}$ - der Platz der Transformation. Anmerkungen zur arabischen

Revolution

Basma El Husseiny

,Transformation und Partnerschaft‘ als kulturpolitische Strategie. Die Rolle des Goethe-Instituts im tunesischen Transformationsprozess

Meike Lettau

\section{Das (Spannungs-)Verhältnis zwischen Kultur und Politik}

Ambivalente Beziehungen. Die Autonomie der Goethe-Institute und die Diplomatie der Deutschen Botschaften

Johannes Crückeberg

Gütesiegel ,Goethe`. Zur Geschichte eines Instituts zwischen Außen- und

Kulturpolitik 95

Bernhard Wittek

Vertrag und Auftrag, Personal und Programm. Das Goethe-Institut zwischen institutionellen und inhaltlichen Widersprüchen Horst Harnischfeger 


\section{Das Goethe-Institut als Akteur von Demokratieförderung}

Am deutschen Wesen soll die Welt nicht genesen! Ein Plädoyer für die internationale kulturelle Zusammenarbeit

Kathinka Dittrich-van Weringh

Für eine demokratische Gesellschaft. Die Kulturarbeit des Goethe-Instituts

in Iran, Brasilien und der Türkei

Kurt Scharf

Wie viel Freiheit braucht Kulturarbeit im Ausland? Programmatische

Projekte mit Partnern des Goethe-Instituts

Michael de la Fontaine

Auf vermintem Gelände. Das Goethe-Institut Chile ermöglicht

Vergangenheitsbewältigung

Dieter Strauss

\section{Fair Cooperation: Austausch auf Augenhöhe}

Anthropophagie als Praxis. Das Goethe-Institut und der kulturelle

Austausch

Bruno Fischli

Kooperationskultur in den Künsten. Perspektiven am Beispiel

deutsch-indischer Partnerschaften

Annika Hampel

Auswärtige Kulturpolitik als Netzwerk. Das Goethe-Institut: Von der

,Einbahnstraße‘ zum ,Global Player`

Hans-Georg Knopp

Die Kunst, Transformationsprozesse zu begleiten. Zur Reform Auswärtiger Kulturpolitik

Wolfgang Schneider und Anna Kaitinnis

Autorenverzeichnis 


\section{Abkürzungsverzeichnis}

$\begin{array}{ll}\text { AKP } & \text { Auswärtige Kulturpolitik } \\ \text { AU } & \text { Afrikanische Union } \\ \text { BMZ } & \begin{array}{l}\text { Bundesministerium für wirtschaftliche Zusammenarbeit und Ent- } \\ \text { wicklung }\end{array} \\ \text { BRD } & \text { Bundesrepublik Deutschland } \\ \text { CDU } & \text { Christlich Demokratische Union Deutschlands } \\ \text { CSU } & \text { Christlich-Soziale Union in Bayern } \\ \text { DAAD } & \text { Deutscher Akademischer Austauschdienst } \\ \text { DCMA } & \text { Dhow Countries Music Academy } \\ \text { DDR } & \text { Deutsche Demokratische Republik } \\ \text { DM } & \text { Deutsche Mark } \\ \text { EU } & \text { Europäische Union } \\ \text { EUNIC } & \text { European Union National Institutes for Culture } \\ \text { FACT } & \text { Forum des Associations Culturelles Tunisiennes } \\ \text { FDP } & \text { Freie Demokratische Partei } \\ \text { HKW } & \text { Haus der Kulturen der Welt } \\ \text { ifa } & \text { Institut für Auslandsbeziehungen } \\ \text { IP } & \text { Interviewpartner (anonymisiert) } \\ \text { IS } & \text { Islamischer Staat } \\ \text { NGO } & \text { Nichtregierungsorganisation } \\ \text { PV } & \text { Pädagogische Verbindungsarbeit } \\ \text { SPD } & \text { Sozialdemokratische Partei Deutschlands } \\ \text { UNESCO } & \text { United Nations Educational, Scientific and Cultural Organization } \\ \text { USA } & \text { United States of America }\end{array}$

